\title{
Analysis of a Co-Epidemic Model
}

\author{
Quinn Morris ${ }^{* \dagger}$ \\ Department of Mathematics, Wake Forest University
}

\begin{abstract}
Solutions to systems of differential equations which model disease transmission are of particular use and importance to epidemiologists who wish to study effective means to slow and prevent the spread of disease. In this paper, we examine a system that models two related diseases within a population, which is of particular importance to those studying co-infection and partial cross-immunity phenomena. Criteria for stability of equilibria are improved upon from previous research by Long, Vaidya, and Brandeau (2008).
\end{abstract}

\section{Introduction}

Since the emergence of AIDS, HIV and tuberculosis have been closely linked. Experiments have indicated that the presence of both HIV and tuberculosis in a population causes increased infectiousness of co-infected persons, and hence there is great interest in studying the epidemiology of such a co-epidemic [3]. In general, a co-epidemic is defined as the related spread of two or more diseases. In a contrasting case, related strains of the influenza virus have been shown to impart some level of cross-immunity, providing further impetus for the study of such epidemics [1]. Despite the importance of such research, little has been done by way of mathematical modeling of such epidemics.

Long, Vaidya, and Brandeau proposed a co-epidemic model in 2008, which modeled the behavior of the coepidemic as a system of nonlinear differential equations [5]. The model is

\footnotetext{
*Funded by Wake Forest University Undergraduate Research Fellowship

${ }^{\dagger}$ Advised by Dr. Stephen B. Robinson, Professor of Mathematics, Wake Forest University
} 


$$
\begin{aligned}
S^{\prime} & =\Lambda-\beta_{1} \frac{S}{N}\left(I_{1}+I_{3}\right)-\tau_{1} \frac{S}{N}\left(I_{2}+I_{3}\right)-\mu S \\
I_{1}^{\prime} & =\beta_{1} \frac{S}{N}\left(I_{1}+I_{3}\right)-\tau_{2} \frac{I_{1}}{N}\left(I_{2}+I_{3}\right)-\left(\mu+\mu_{1}\right) I_{1} \\
I_{2}^{\prime} & =\tau_{1} \frac{S}{N}\left(I_{2}+I_{3}\right)-\beta_{2} \frac{I_{2}}{N}\left(I_{1}+I_{3}\right)-\left(\mu+\mu_{2}\right) I_{2} \\
I_{3}^{\prime} & =\beta_{2} \frac{I_{2}}{N}\left(I_{1}+I_{3}\right)+\tau_{2} \frac{I_{1}}{N}\left(I_{2}+I_{3}\right)-\left(\mu+\mu_{1}+\mu_{2}\right) I_{3} \\
N^{\prime} & =\Lambda-\mu N-\mu_{1} I_{1}-\mu_{2} I_{2}-\left(\mu_{1}+\mu_{2}\right) I_{3},
\end{aligned}
$$

where $S$ represents the number of susceptible people in the population, $I_{1}$ and $I_{2}$ represent the number of people infected with disease 1 and disease 2, respectively, $I_{3}$ represents the number of people infected with diseases 1 and 2 , and $N=S+I_{1}+I_{2}+I_{3}$ is the total population. The recruitment function, $\Lambda$, will be assumed to be a positive constant which represents entrance into the susceptible population, $S$, either through birth, as would be the case with influenza, or through entrance into the sexually-active population as with sexually transmitted diseases.

The parameters $\beta_{i}$ and $\tau_{i}$, with $i=1,2$, represent the contact rates for disease 1 and disease 2 , respectively. The model does not, however, allow for a susceptible individual to catch both disease 1 and disease 2 simultaneously, and hence there is no term to represent transfer from $S$ to $I_{3}$ directly. It is also possible that from each class, an individual may die of natural causes (those not related to any disease), and this happens at a rate $\mu$. Furthermore, those infected with disease 1 or disease 2 may die from the disease at a rate of $\mu_{1}$ or $\mu_{2}$, respectively. This assumption, the fact that we do not allow for recovery and reentry into the susceptible population, and the fact that member of the $I_{3}$ class are able to spread diseases 1 and 2 at the same rate are fairly strong assumptions that the model makes about disease transmission, which simplify the dynamics quite a bit.

All the given constants have epidemiological significance, and perhaps the most epidemiologically significant term is the basic reproductive number of disease $i, R_{0}^{i}$, which for our diseases is defined as $R_{0}^{1}=\frac{\beta_{1}}{\mu+\mu_{1}}$ and $R_{0}^{2}=\frac{\tau_{1}}{\mu+\mu_{2}}$. This ratio, of the contact rate to the total death rate from the infected class, is used to determine whether the disease will persist in the long run. If the disease is much more contagious than it is deadly, then a large percentage of the population will become infected. If, on the other hand, the disease is much more deadly than it is contagious, then people who catch the disease 
will die before being able to infect anyone else. In general, if $R_{0}^{i}<1$, then the disease will die out, while if $R_{0}^{i}>1$, the disease will persist. Given the idea of basic reproductive number, we wish to define similar quantities for the co-epidemic model, and find similar persistence criteria. In this paper, we establish more precise and, in some cases, new criteria for existence and stability of system equilibria.

\section{Stability of the Disease-Free Equilibrium}

The disease-free equilibrium is defined as the point at which no disease is present in the population, which is represented in the model as $I_{1}=I_{2}=$ $I_{3}=0$. The system of equations simplifies to

$$
\begin{aligned}
& S^{\prime}=\Lambda-\mu S \\
& I_{1}^{\prime}=0 \\
& I_{2}^{\prime}=0 \\
& I_{3}^{\prime}=0 .
\end{aligned}
$$

Thus, the disease-free equilibrium lies at the point $\left(\frac{\Lambda}{\mu}, 0,0,0\right)$.

Using a Lyapunov function, Long, Vaidya, and Brandeau showed that the disease-free equilibrium is globally asymptotically stable if

$$
\max \left\{R_{0}^{1}, R_{0}^{2}, \frac{\beta_{1}+\tau_{1}}{\mu+\mu_{1}+\mu_{2}}\right\}<\frac{1}{2} .
$$

However, many other standard models of disease transmission have a globally asymptotically stable equilibrium if $\max \left\{R_{0}^{1}, R_{0}^{2}\right\}<1$ (cf. SIS and SEIR models in [2]). We therefore strive to find a stronger result.

Theorem 2.1 The disease-free equilibrium is globally asymptotically stable if $\max \left\{R_{0}^{1}, R_{0}^{2}, \frac{\beta_{1}+\tau_{1}}{\mu+\mu_{1}+\mu_{2}}\right\}<1$.

In order to prove this theorem, we first establish a number of claims:

1. Solution curves with all nonnegative initial conditions never have a term which becomes negative, i.e., the "positive cone" is invariant.

2. $\left(I_{1}+I_{2}+I_{3}\right)^{\prime}<0$.

3. $\left(I_{1}+I_{2}+I_{3}\right) \rightarrow 0$ as $t \rightarrow \infty$.

4. $S \rightarrow \frac{\Lambda}{\mu}$ as $t \rightarrow \infty$. 


\subsection{Claim 1: The Positive Cone is Invariant}

In order to show that solution curves with nonnegative initial conditions remain in the positive cone (the region of the solution space with $S, I_{1}, I_{2}, I_{3} \geq$ 0 ), we wish to examine behavior of solution curves with initial conditions on the boundary of the positive cone. If we can show that these solution curves either stay on the boundary or bounce into the interior of the positive cone, then we have shown that the positive cone is invariant.

Let us begin with the simplest boundary case with initial condition $\left(S^{0}, 0,0,0\right)$. The system of equations simplifies to (2). In this case, the explicit solution is $S=c e^{\mu t}-\frac{\Lambda}{\mu}$ with all other terms identically zero. It follows that any solution curve with a point on the $S$-axis must lie entirely on the $S$-axis. Moreover, if $S^{0}>0$, then $S$ stays positive.

Now, let us consider a more complex set of initial conditions. Let $\left(S^{0}, I_{1}^{0}, 0,0\right)$ be an initial condition for the system with $I_{1}^{0}>0$ and $S^{0} \geq 0$. Note that by substituting this initial condition into (1), the system reduces to

$$
\begin{aligned}
& S^{\prime}=\Lambda-\beta_{1} \frac{S}{N} I_{1}-\mu S \\
& I_{1}^{\prime}=\beta_{1} \frac{S}{N} I_{1}-\left(\mu+\mu_{1}\right) I_{1} \\
& I_{2}^{\prime}=0 \\
& I_{3}^{\prime}=0 .
\end{aligned}
$$

The first two equations do not depend on $I_{2}$ or $I_{3}$, so it follows from the existence and uniqueness theorem that the solutions are contained entirely within the $S-I_{1}$ plane. We wish to show that solutions remain within the first quadrant of the $S-I_{1}$ plane. By the previous argument, the solution cannot contain a point where $I_{1}=0$, so $I_{1}(t)>0$ for all $t$. Any solution curve which touches the $S=0$ boundary must be immediately bounced back into the first quadrant, as $S^{\prime}=\Lambda$ along the $S=0$ boundary. Hence, all solutions with initial conditions of this form remain in the positive cone. An analogous argument can be made for initial conditions of the form $\left(S^{0}, 0, I_{2}^{0}, 0\right)$.

For initial conditions of the form $\left(S^{0}, 0,0, I_{3}^{0}\right)$ with $S^{0}, I_{3}^{0}>0$, the system simplifies to

$$
\begin{aligned}
& S^{\prime}=\Lambda-\beta_{1} \frac{S}{N}\left(I_{3}\right)-\tau_{1} \frac{S}{N}\left(I_{3}\right)-\mu S \\
& I_{1}^{\prime}=\beta_{1} \frac{S}{N}\left(I_{3}\right) \\
& I_{2}^{\prime}=\tau_{1} \frac{S}{N}\left(I_{3}\right) \\
& I_{3}^{\prime}=\beta_{2} \frac{I_{2}}{N}\left(I_{1}+I_{3}\right)+\tau_{2} \frac{I_{1}}{N}\left(I_{2}+I_{3}\right)-\left(\mu+\mu_{1}+\mu_{2}\right) I_{3} .
\end{aligned}
$$


Though $S$ and $I_{3}$ may be decreasing, we can choose a sufficiently small time step, $\delta$, such that $S(t), I_{3}(t)>0$ for $0<t<\delta$. Since $I_{1}^{\prime}(0), I_{2}^{\prime}(0)>0$, we can also choose $\delta$ such that $I_{1}(t), I_{2}(t) \geq 0$ for $0<t<\delta$. The solution curve therefore remains in the positive cone. If $S^{0}=0$ and $I_{3}^{0}>0$, then a similar argument follows for showing that the solution curve is entirely within the positive cone.

For initial conditions of the form $\left(0, I_{1}^{0}, I_{2}^{0}, 0\right),\left(0, I_{1}^{0}, 0, I_{3}^{0}\right)$, $\left(0,0, I_{2}^{0}, I_{3}^{0}\right)$, and $\left(S^{0}, I_{1}^{0}, I_{2}^{0}, 0\right)$, a similar argument can be made, showing that for these initial conditions, solutions begin on the boundary, but are forced off into the positive cone.

Therefore, for any set of initial conditions $\left(S^{0}, I_{1}^{0}, I_{2}^{0}, I_{3}^{0}\right)$ such that $S^{0}, I_{1}^{0}, I_{2}^{0}, I_{3}^{0} \geq$ 0 , the solution curve $\left(S(t), I_{1}(t), I_{2}(t), I_{3}(t)\right) \geq 0$. Hence, all solution curves with non-negative initial conditions, which are the only ones of epidemiological significance, remain in the positive cone. Thus, we have shown the following lemma:

Lemma 2.2 Given a set of initial conditions $\left(S^{0}, I_{1}^{0}, I_{2}^{0}, I_{3}^{0}\right)$ in the positive cone, the solution curve through the point remains in the positive cone.

\subsection{Claim 2: $\left(I_{1}+I_{2}+I_{3}\right)^{\prime}<0$}

Consider the quantity $\left(I_{1}+I_{2}+I_{3}\right)^{\prime}$.

$$
\begin{aligned}
\left(I_{1}+I_{2}+I_{3}\right)^{\prime}= & I_{1}^{\prime}+I_{2}^{\prime}+I_{3}^{\prime} \\
= & \beta_{1} \frac{S}{N}\left(I_{1}+I_{3}\right)+\tau_{1} \frac{S}{N}\left(I_{2}+I_{3}\right) \\
& -\left(\mu+\mu_{1}\right) I_{1}-\left(\mu+\mu_{2}\right) I_{2}-\left(\mu+\mu_{1}+\mu_{2}\right) I_{3} \\
= & \left(\beta_{1} \frac{S}{N}-\left(\mu+\mu_{1}\right)\right) I_{1}+\left(\tau_{1} \frac{S}{N}-\left(\mu+\mu_{2}\right)\right) I_{2} \\
& +\left(\left(\beta_{1}+\tau_{1}\right) \frac{S}{N}-\left(\mu+\mu_{1}+\mu_{2}\right)\right) I_{3} \\
\leq & \left(\beta_{1}-\left(\mu+\mu_{1}\right)\right) I_{1}+\left(\tau_{1}-\left(\mu+\mu_{2}\right)\right) I_{2} \\
& +\left(\left(\beta_{1}+\tau_{1}\right)-\left(\mu+\mu_{1}+\mu_{2}\right)\right) I_{3} \\
\leq & -\epsilon\left(I_{1}+I_{2}+I_{3}\right)
\end{aligned}
$$

where $\epsilon=\max \left\{\beta_{1}-\left(\mu+\mu_{1}\right), \tau_{1}-\left(\mu+\mu_{2}\right),\left(\beta_{1}+\tau_{1}\right)-\left(\mu+\mu_{1}+\mu_{2}\right)\right\}>0$ if $\max \left\{R_{0}^{1}, R_{0}^{2}, \frac{\beta_{1}+\tau_{1}}{\mu+\mu_{1}+\mu_{2}}\right\}<1$. This implies that the solution curve is bounded above by a solution that decays exponentially to zero. Thus we have shown the following lemma:

Lemma 2.3 If $\max \left\{R_{0}^{1}, R_{0}^{2}, \frac{\beta_{1}+\tau_{1}}{\mu+\mu_{1}+\mu_{2}}\right\}<1$ and if $\left(I_{1}, I_{2}, I_{3}\right) \neq(0,0,0)$ then the number of infected persons is everywhere decreasing. 
2.3 Claim 3: $\left(I_{1}+I_{2}+I_{3}\right) \rightarrow 0$ as $t \rightarrow \infty$

A simple extension of the proof above will prove this lemma. Since $I_{1}+I_{2}+$ $I_{3} \leq-\epsilon\left(I_{1}+I_{2}+I_{3}\right)^{\prime}$, then the sum must be decreasing until such a time as $I_{1}+I_{2}+I_{3}=0$. Since $0 \leq I_{1}, I_{2}, I_{3}$, each must be approaching zero in the limit. Hence, as $t$ approaches infinity, the number of infective people in each class must approach 0 . Thus we have shown the following lemma:

Lemma 2.4 As $t \rightarrow \infty, I_{1}, I_{2}, I_{3} \rightarrow 0$.

\subsection{Claim 4: $S \rightarrow \frac{\Lambda}{\mu}$ as $t \rightarrow \infty$}

Given Lemma 2.4, we know that for any $\delta>0$, there exists $T>0$ such that $\left|\beta_{1} \frac{S}{N}\left(I_{1}+I_{3}\right)+\tau_{1} \frac{S}{N}\left(I_{2}+I_{3}\right)\right|<\frac{\delta}{2}$ for all $t \geq T$. If we choose $S \geq \frac{\Lambda+\delta}{\mu}$, then $\Lambda-\mu S \leq-\delta$. Hence, $S^{\prime}=\left(\beta_{1} \frac{S}{N}\left(I_{1}+I_{3}\right)+\tau_{1} \frac{S}{N}\left(I_{2}+I_{3}\right)\right)+(\Lambda-\mu S) \leq-\frac{\delta}{2}$. It follows that for any solution, there exists $T>0$ such that $S \leq \frac{\Lambda+\delta}{\mu}$ for all $t>T$. A similar argument follows for $S \geq \frac{\Lambda-\delta}{\mu}$. It follows that $S \rightarrow \frac{\Lambda}{\mu}$.

Lemma 2.5 The number of susceptibles approaches the equilibrium value $S=\frac{\Lambda}{\mu}$ as $t \rightarrow \infty$, i.e., the disease free equilibrium is globally asymptotically stable.

\section{Stability of the Quasi-Disease-Free Equilib- ria}

Expanding our view outside the realm of the disease-free equilibrium, we now turn our interest to the quasi-disease-free equilibria, where one, but not both, disease is allowed to persist. Arguments for the first and second quasi-diseasefree equilibria are almost identical, so here we only concern ourselves with analysis of the first quasi-disease-free equilibrium. In general, it is difficult to construct a local or global stability argument for the quasi-disease-free equilibria. Where possible, the system will be left as in (1), however at times, certain assumptions must be made in order to simplify the analysis.

\subsection{Long-term Behavior of Solutions}

Similar to the disease-free-equilibrium, we can expect that the sum of the disease classes for which $R_{0}<1$ will tend to zero. In the case of the first 
quasi-disease-free equilibrium, this means that we expect $I_{2}+I_{3} \rightarrow 0$ as $t \rightarrow \infty$. We first show that $\left(I_{2}+I_{3}\right)^{\prime}<0$ if $\tau_{i} \leq \mu+\mu_{2}$ for $i=1,2$.

$$
\begin{aligned}
\left(I_{2}+I_{3}\right)^{\prime} & =\tau_{1} \frac{S}{N}\left(I_{2}+I_{3}\right)-\left(\mu+\mu_{2}\right) I_{2}+\tau_{2} \frac{I_{1}}{N}\left(I_{2}+I_{3}\right)-\left(\mu+\mu_{1}+\mu_{2}\right) I_{3} \\
& =\left[\left(\tau_{1} \frac{S}{N}+\tau_{2} \frac{I_{1}}{N}\right)-\left(\mu+\mu_{2}\right)\right]\left(I_{2}+I_{3}\right)-\mu_{1} I_{3} \\
& <-\epsilon\left(I_{2}+I_{3}\right) \\
& <0
\end{aligned}
$$

where $\epsilon \leq\left(\mu+\mu_{2}\right)-\tau_{i}$ for $i=1,2$.

As in Section 2, we can now use this fact to show that for any value of $I_{2}+I_{3}$ greater than zero, the sum approaches 0 .

Using this information, we now know that the long-term behavior of the system will be the same as the behavior on the $S-I_{1}$ plane [8]. Having reduced the problem to a two-dimensional problem, we can apply the PoincaréBendixson Theorem.

\subsection{Global Stability in the $S-I_{1}$ plane and the Poincaré- Bendixson Theorem}

For this section, let the system be described as follows in the $S-I_{1}$ plane.

$$
\begin{aligned}
& S^{\prime}=f\left(S, I_{1}\right)=\Lambda-\beta_{1} \frac{S}{N} I_{1}-\mu S \\
& I_{1}^{\prime}=g\left(S, I_{1}\right)=\beta_{1} \frac{S}{N} I_{1}-\left(\mu+\mu_{1}\right) I_{1}
\end{aligned}
$$

By setting both equations equal to zero and solving them simultaneously, we find that the quasi-disease-free equilibrium is $\left(S^{*}, I_{1}^{*}\right)=\left(\frac{\Lambda}{\beta_{1}-\mu_{1}}, \frac{\Lambda\left(R_{0}^{1}-1\right)}{\beta_{1}-\mu_{1}}\right)$, where $R_{0}^{1}=\frac{\beta_{1}}{\mu+\mu_{1}}$. Note that this equilibrium exists in the positive cone for $R_{0}^{1}>1$. Now, we look at the Jacobian of the system at the equilibrium in order to determine stability.

$$
D_{\vec{x}} \vec{F}\left(S, I_{1}\right)=\left(\begin{array}{cc}
-\frac{\beta_{1} I_{1}^{2}}{N^{2}}-\mu & -\frac{\beta_{1} S^{2}}{N^{2}} \\
\frac{\beta_{1} I_{1}^{2}}{N^{2}} & \frac{\beta_{1} S^{2}}{N^{2}}-\left(\mu+\mu_{1}\right)
\end{array}\right) .
$$

Computing the trace of this matrix evaluated at the equilibrium $\left(S^{*}, I_{1}^{*}\right)$, we 
see that

$$
\begin{aligned}
\operatorname{tr}\left(D_{\vec{x}} \vec{F}\left(S^{*}, I_{1}^{*}\right)\right) & =-\frac{\beta_{1}\left(I_{1}^{*}\right)^{2}}{\left(N^{*}\right)^{2}}-\mu+\frac{\beta_{1}\left(S^{*}\right)^{2}}{\left(N^{*}\right)^{2}}-\left(\mu+\mu_{1}\right) \\
& =\frac{\beta_{1}}{\left(R_{0}^{1}\right)^{2}}\left(1-\left(R_{0}^{1}-1\right)^{2}\right)-2 \mu-\mu_{1} \\
& =\frac{\beta_{1}}{\left(R_{0}^{1}\right)^{2}}\left(2 R_{0}^{1}-\left(R_{0}^{1}\right)^{2}\right)-2 \mu-\mu_{1} \\
& =\left(\mu+\mu_{1}\right)\left(2-R_{0}^{1}\right)-2 \mu-\mu_{1} \\
& =\left(\mu+\mu_{1}\right)\left(1-R_{0}^{1}\right)-\mu \\
& <0
\end{aligned}
$$

since $R_{0}^{1}>1$.

By replacing the second row with the sum of the two rows and computing the determinant of this matrix evaluated at the equilibrium $\left(S^{*}, I_{1}^{*}\right)$, we find

$$
\begin{aligned}
\operatorname{det}\left(D_{\vec{x}} \vec{F}\left(S^{*}, I_{1}^{*}\right)\right) & =\left(\frac{\beta_{1}\left(R_{0}^{1}-1\right)^{2}}{\left(R_{0}^{1}\right)^{2}}+\mu\right)\left(\frac{\beta_{1}}{R_{0}^{1}}\right)-\frac{\mu \beta_{1}}{\left(R_{0}^{1}\right)^{2}} \\
& =\left(R_{0}^{1}-1\right) \frac{\beta_{1}}{R_{0}^{1}}\left(\frac{\beta_{1}\left(R_{0}^{1}+1\right)+\mu R_{0}^{1}}{\left(R_{0}^{1}\right)^{2}}\right) \\
& >0
\end{aligned}
$$

for $R_{0}^{1}>1$.

Given that $\operatorname{det}\left(D_{\vec{x}} \vec{F}\left(S^{*}, I_{1}^{*}\right)\right)>0$ and $\operatorname{tr}\left(D_{\vec{x}} \vec{F}\left(S^{*}, I_{1}^{*}\right)\right)<0$, we conclude that the eigenvalues, $\lambda_{1}, \lambda_{2}$, of $D_{\vec{x}} \vec{F}\left(S^{*}, I_{1}^{*}\right)$ both have negative real part, and hence the equilibrium is locally stable for $R_{0}^{1}>1$ and $R_{0}^{2}<1$.

It is worth noting here that this condition for local stability is an improvement upon that of Long, Vaidya, and Brandeau, who showed in 2008 that the first quasi-disease-free equilibrium is locally stable for $R_{0}^{1}>1, R_{0}^{2}<1$, and $\mu+\mu_{1}+\mu_{2}>\tau\left[1+\frac{\mu_{1}}{R_{0}^{1}\left(\beta-\mu_{1}+\mu_{2}\right)}\right]$ where it is assumed that $\beta=\beta_{1}=\beta_{2}$ and $\tau=\tau_{1}=\tau_{2}$.

Given the the local stability of the equilibrium and the boundedness of trajectories of the system, we can apply the Poincaré-Bendixson theorem. To show that the equilibrium is stable, we must rule out the possibility of a periodic trajectory or a trajectory whose $\omega$-limit set contains the disease-free equilibrium. Using the Bendixson-Dulac criterion, we can rule out a periodic orbit if we can find a function, $\alpha\left(S, I_{1}\right)$, such that $\frac{\partial}{\partial S}(\alpha f)+\frac{\partial}{\partial I_{1}}(\alpha g)$ does not change sign and is not identically zero on any open set in the $S-I$ plane [7]. If we choose $\alpha\left(S, I_{1}\right)=\frac{1}{S I_{1}}$, then $\frac{\partial}{\partial S}(\alpha f)+\frac{\partial}{\partial I_{1}}(\alpha g)=\frac{-\Lambda}{\left(S+I_{1}\right)^{2}}$, which does not change sign on any interval since $\Lambda$ is constant. Therefore, we have ruled out the possibility of a periodic trajectory. We can rule out the possibility that the $\omega$-limit contains the disease-free equilibrium by application of Lemma 2.2 in [8], since the disease-free equilibrium is a saddle. Hence, the quasi-diseasefree equilibrium is globally asymptotically stable for the given conditions. 


\section{Existence of a Co-infection Equilibrium}

In this section, we rely on bifurcation results from [6] to determine the existence of a co-infection equilibrium. While previous arguments have relied almost solely on using algebra and calculus to simplify things to twodimensional problems, in this section, we will explicitly compute a number of quantities related to the system. For ease of notation, let $f_{1}, f_{2}, f_{3}$, and $f_{4}$ represent the right-hand sides of $S^{\prime}, I_{1}^{\prime}, I_{2}^{\prime}$, and $I_{3}^{\prime}$, respectively, let $\vec{F}=\left(\begin{array}{c}f_{1} \\ f_{2} \\ f_{3} \\ f_{4}\end{array}\right)$ and let $\vec{x}=\left(\begin{array}{c}S \\ I_{1} \\ I_{2} \\ I_{3}\end{array}\right)$.

We wish to show that a bifurcation to a co-infection equilibrium exists with bifurcation parameter $\tau_{2}$, where $F\left(\tau_{2}, \vec{x}^{*}\right)=0$ for all $\tau_{2}$ and

$$
\vec{x}^{*}=\left(\begin{array}{c}
S^{*} \\
I_{1}^{*} \\
0 \\
0
\end{array}\right) .
$$

There are two conditions that must be shown.

1. $D_{\vec{x}} \vec{F}$ has a one-dimensional null space.

2. $D_{\vec{x} \tau_{2}} \vec{F} \cdot \vec{u}_{0}$, is not in the range of $D_{\vec{x}} \vec{F}$, where $\vec{u}_{0}$ is the left null vector of $D_{\vec{x}} \vec{F}$.

\subsection{Dimension of the null space of $D_{\vec{x}} \vec{F}$}

In order to show the first condition, we first compute the matrix $D_{\vec{x}} \vec{F}$.

$$
D_{\vec{x}} \vec{F}=\left(\begin{array}{cc}
\mathbf{A}_{1,1} & \mathbf{A}_{1,2} \\
\mathbf{A}_{2,1} & \mathbf{A}_{2,2}
\end{array}\right)
$$

where

$$
\mathbf{A}_{\mathbf{1}, \mathbf{1}}=\left(\begin{array}{cc}
-\frac{\beta_{1} I_{1}}{S+I_{1}}+\frac{\beta_{1} S I_{1}}{\left(S+I_{1}\right)^{2}}-\mu & -\frac{\beta_{1} S}{S+I_{1}}+\frac{\beta_{1} S I_{1}}{\left(S+I_{1}\right)^{2}} \\
\frac{\beta_{1} I_{1}}{S+I_{1}}-\frac{\beta_{1} S I_{1}}{\left(S+I_{1}\right)^{2}} & \frac{\beta_{1} S}{S+I_{1}}-\frac{\beta_{1} S I_{1}}{\left(S+I_{1}\right)^{2}}-\mu-\mu_{1}
\end{array}\right),
$$




$$
\begin{gathered}
\mathbf{A}_{\mathbf{1 , 2}}=\left(\begin{array}{cc}
\frac{\beta_{1} S I_{1}}{\left(S+I_{1}\right)^{2}}-\frac{\tau_{1} S}{S+I_{1}} & -\frac{\beta_{1} S}{S+I_{1}}+\frac{\beta_{1} S I_{1}}{\left(S+I_{1}\right)^{2}}-\frac{\tau_{1} S}{S+I_{1}} \\
-\frac{\beta_{1} S I_{1}}{\left(S+I_{1}\right)^{2}}-\frac{\tau_{2} I_{1}}{S+I_{1}} & \frac{\beta_{1} S}{S+I_{1}}-\frac{\beta_{1} S I_{1}}{\left(S+I_{1}\right)^{2}}-\frac{\tau_{2} I_{1}}{S+I_{1}}
\end{array}\right), \\
\mathbf{A}_{\mathbf{2}, \mathbf{1}}=\left(\begin{array}{cc}
0 & 0 \\
0 & 0
\end{array}\right), \\
\mathbf{A}_{\mathbf{2}, \mathbf{2}=}=\left(\begin{array}{cc}
\frac{\tau_{1} S}{S+I_{1}}-\frac{\beta_{2} I_{1}}{S+I_{1}}-\mu-\mu_{2} & \frac{\tau_{1} S}{S+I_{1}} \\
\frac{\beta_{2} I_{1}}{S+I_{1}}+\frac{\tau_{2} I_{1}}{S+I_{1}} & \frac{\tau_{2} I_{1}}{S+I_{1}}-\mu-\mu_{1}-\mu_{2}
\end{array}\right) .
\end{gathered}
$$

Since $D_{\vec{x}} \vec{F}$ has a block structure that is upper-triangular, we focus our analysis on the submatrices $\mathbf{A}_{\mathbf{1 , 1}}$ and $\mathbf{A}_{\mathbf{2}, \mathbf{2}}$. For submatrix $\mathbf{A}_{\mathbf{1 , 1}}$, we wish to show that it has a non-zero, positive determinant. We notice here that $\mathbf{A}_{\mathbf{1 , 1}}$ is exactly the same matrix from section $3.2, \operatorname{so} \operatorname{det}\left(\mathbf{A}_{\mathbf{1}, \mathbf{1}}\right)>0$ We then wish to show that for some specific value of $\tau_{2}=\tau_{2}^{*}$, $\operatorname{det}\left(\mathbf{A}_{\mathbf{2}, \mathbf{2}}\right)=0$. Calculating $\operatorname{det}\left(\mathbf{A}_{\mathbf{2}, \mathbf{2}}\right)$, we see that it is linear in $\tau_{2}$.

$$
\begin{aligned}
\operatorname{det}\left(\mathbf{A}_{\mathbf{2}, 2}\right)=\left(\frac{\tau_{1} S}{S+I_{1}}-\frac{\beta_{2} I_{1}}{S+I_{1}}-\mu-\mu_{2}\right) & \left(\frac{\tau_{2} I_{1}}{S+I_{1}}-\mu-\mu_{1}-\mu_{2}\right) \\
& -\left(\frac{\tau_{1} S}{S+I_{1}}\right)\left(\frac{\beta_{2} I_{1}}{S+I_{1}}+\frac{\tau_{2} I_{1}}{S+I_{1}}\right) .
\end{aligned}
$$

Hence, for some value of $\tau_{2}=\tau_{2}^{*}, \mathbf{A}_{\mathbf{2 , 2}}$ is a nontrivial matrix with $\operatorname{det}\left(\mathbf{A}_{\mathbf{2}, \mathbf{2}}\right)=0$ and $D_{\vec{x}} \vec{F}$ has a one-dimensional null space. Note that as $\tau_{2}$ transitions from smaller to larger, $\operatorname{det}\left(\mathbf{A}_{\mathbf{2}, \mathbf{2}}\right)$ transitions from positive to negative. At $\tau_{2}=\tau_{2}^{*}$, we have one negative eigenvalue and a maximum eigenvalue of zero. It follows by continuity that for $\tau_{2}$ near $\tau_{2}^{*}$ the eigenvalues are real and distinct with at least one of them being negative. Since $\operatorname{det}\left(\mathbf{A}_{\mathbf{2}, \mathbf{2}}\right)$ transitions from positive to negative, it must be the case that the maximum eigenvalue changes from negative to positive as $\tau_{2}$ increases through $\tau_{2}^{*}$. The maximum eigenvalue of this matrix will now be referred to as $\gamma\left(\tau_{2}\right)$, and we have thus far established that $\gamma^{\prime}\left(\tau_{2}^{*}\right)>0$.

\subsection{The range of $D_{\vec{x}} \vec{F}$}

We now wish to show that the null vector of $D_{\vec{x} \tau_{2}} \vec{F} \cdot u_{0}$ is not in the range of $D_{\vec{x}} \vec{F}$. First, we calculate $D_{\vec{x} \tau_{2}} \vec{F}$. 


$$
D_{\vec{x} \tau_{2}} \vec{F}=\left(\begin{array}{cccc}
0 & 0 & 0 & 0 \\
0 & 0 & -\frac{I_{1}}{S+I_{1}} & -\frac{I_{1}}{S+I_{1}} \\
0 & 0 & 0 & 0 \\
0 & 0 & \frac{I_{1}}{S+I_{1}} & \frac{I_{1}}{S+I_{1}}
\end{array}\right) .
$$

Hence, any vector in the range of $D_{\vec{x} \tau_{2}} \vec{F}$, in particular $D_{\vec{x} \tau_{2}} \vec{F} \cdot u_{0}$, must be of the form

$$
c\left(\begin{array}{c}
0 \\
-1 \\
0 \\
1
\end{array}\right)
$$

where $c$ is a constant.

We represent the range of $D_{\vec{x} \tau_{2}} \vec{F}$ with the vector above, and show that the matrix $D_{\vec{x}} \vec{F}$ has no vector in its range of the above form.

Since $D_{\vec{x}} \vec{F}$ has block structure, we need only show that the equation below has no solution.

$$
\mathbf{A}_{2,2}\left(\begin{array}{l}
x_{1} \\
x_{2}
\end{array}\right)=\left(\begin{array}{l}
0 \\
1
\end{array}\right) .
$$

Since we chose $\tau_{2}=\tau_{2}^{*}$, then $\operatorname{det}\left(\mathbf{A}_{\mathbf{2}, \mathbf{2}}\right)=0$, and it follows that there is no vector which satisfies the above equation because the rows of $\mathbf{A}_{\mathbf{2 , 2}}$ are linearly dependent and the first row is not identically zero. Hence, $D_{\vec{x} \tau_{2}} \vec{F} \cdot \vec{u}_{0}$, is not in the range of $D_{\vec{x}} \vec{F}$.

We therefore conclude that there exists some range of $\tau_{2}$ on which there exists a non-trivial bifurcation curve, which can be described as $\left(\tau_{2}, \vec{x}\right)=$ $\left(\tau_{2}^{*}+s \tau_{2}^{\prime}(0)+o(s), \vec{x}^{*}+s \vec{u}_{0}+o(s)\right.$, where $s$ is the variable of parameterization and

$$
\vec{u}_{0}=\left(\begin{array}{c}
\cdot \\
\cdot \\
+ \\
+
\end{array}\right) \text {. }
$$

For specific parameters $\left(\beta_{1}=\beta_{2}=\tau_{1}=\frac{1}{2}\right.$ and $\left.\mu=\mu_{1}=\mu_{2}=\frac{1}{10}\right)$, it can be shown numerically that the bifurcation is to a stable co-infection equilibrium, but a proof that the bifurcation is to a stable co-infection equilibrium for a certain set of parameters has not yet been found. 


\section{$5 \quad$ Further Research}

Though significant inroads have been made toward finding sharp conditions for stability, there are many opportunities for further research into the precision of stability criteria for quasi-disease-free and co-infection equilibria. The question as to the stability of the coinfection equilibria remains open. In addition to general calculation, numerical simulation of several cases would provide insight for further research.

The question that nearly every model presents is how it might be made more realistic while remaining sufficiently manageable to compute. The model used here may be altered to include recovery, with full, partial, or no immunity, or to include a recruitment function, $\Lambda\left(I_{1}\right), \Lambda\left(I_{2}\right)$, or $\Lambda\left(I_{3}\right)$ rather than a recruitment constant. Such alterations would make the model more realistic for certain diseases, in particularly sexually transmitted diseases, where the number of people entering the sexually active population may vary depending on the current rate of STD infection [4].

\section{References}

[1] M. Boni, J. Gog, et. al., Influenza drift and epidemic size: the race between generating and escaping immunity, Theoretical Population Biology, 65 2004, pp. 179-191.

[2] F. Brauer, P. van den Driessche, and J. Wu, eds, Mathematical Epidemiology. Berlin, Springer, 2008.

[3] A. Escombe, D. Moore, et al, The Infectiousness of Tuberculosis Patients Coinfected with HIV, PLoS Med, 5 2008, pp. 1387-1397.

[4] E. Ferry, "Population Dynamics and Sexually Transmitted Diseases", Thesis, Wake Forest University, 1999.

[5] E. Long, N. Vaidya, M. Brandeau, Controlling Co-Epidemics: Analysis of HIV and Tuberculosis Infection Dynamics, Oper. Res, 56 2008, pp. 1366-1381.

[6] J. Smoller, Shock Waves and Reaction-diffusion Equations, New York, Springer-Verlag, 1994. 
[7] P. Waltman. "Limit Cycles and the Poincare-Bendixson Theorem", A Second Course in Elementary Differential Equations, Mineola, NY, Dover Publications, 2004, pp. 141-47.

[8] P. Waltman. Persistence in Models of Three Interacting Predator-Prey Populations, Math. Biosciences 68 1984, pp. 213-231 